Revista Internacional de Sociología RIS

\section{EL POPULISMO: DE INTRUSO A PROBLEMA RELEVANTE PARA LA CIENCIA SOCIAL}

\author{
ARTURo Rodríguez SÁEZ \\ Universidad Complutense de Madrid \\ arsaez@ucm.es \\ ORCID iD: https://orcid.org/0000-0003-0040-5297
}

Cómo citar este artículo / Citation: Rodríguez Sáez, A. 2018. "El populismo: de intruso a problema relevante para la ciencia social". Revista Internacional de Sociología 76(4): e114. https://doi.org/10.3989/ris.2018.76.4.18.076

\section{POPULISM: FROM INTRUDER TO A RELEVANT PROBLEM TO SOCIAL SCIENCE}

Copyright: () 2018 CSIC. Este es un artículo de acceso abierto distribuido bajo los términos de la licencia de uso y distribución Creative Commons Reconocimiento 4.0 Internacional (CC BY 4.0).

Recibido: 09/05/2018. Aceptado: 03/09/2018

\section{Resumen}

Esta nota de investigación destaca la importancia del fenómeno populista para el análisis social y por qué es necesario ir más allá de considerarlo como un intruso de la democracia. Después, se analizan tres libros recientes sobre el fenómeno populista destacando sus diferencias en cuanto a la génesis, naturaleza y consecuencias políticas, así como sus limitaciones al no considerar con la necesaria profundidad sus raíces sociales e históricas. Se concluye señalando que el fenómeno populista es intrínseco a la democracia y expresión de cambios complejos relacionados con períodos de transición hacia nuevas formas de producción, consumo y modos de vida a las que los sistemas democráticos no dan respuesta efectiva, debido a inercias o bloqueos institucionales.

\section{Palabras Clave}

Democracia; Discurso; Ideología; Neoliberalismo; Retórica.

\section{Abstract}

This research note emphasizes the importance of populist phenomenon for social analysis and why is necessary to go beyond its consideration as an intruder of democracy. Further, three recent books on populist phenomenon are revised highlighting their differences related to its foundations, social nature and political consequences, as well as its limitations by not considering with necessary depth its sociological and historical roots. It is concluded that the populist phenomenon is intrinsic to democracy, a consequence of complex changes related to historical periods of transition to new ways of production, consumption and life styles to whom the democratic systems do not give effective answer because of institutional inertias or blockages.

\section{KEYWORDS}

Democracy; Discourse; Ideology; Neoliberalism; Rhetoric. 


\section{INTRODUCCIÓN}

El populismo es un concepto que se halla en los confines de la teoría social y política. Habita en los márgenes, es periférico y retorna siempre. Es un concepto límite para tiempos límite ${ }^{1}$.

La palabra o categoría política populista concita todo tipo de descalificaciones o expresiones negativas (Muddle y Rovira Kaltwasser 2017) por parte de no pocos medios de comunicación y por los propios partidos políticos que lo utilizan como arma arrojadiza en la lucha ideológica. Sólo en el mundo académico cuenta con una importante consideración, a pesar de la dificultad que entraña su análisis y comprensión.

Al populismo suele acompañarle un reduccionismo semántico que tiene como finalidad volver maldito el término. Tal reduccionismo acumula un sinfín de tópicos que se sintetizan en la palabra demagogia como equivalente del populismo, ahorrándose el esfuerzo analítico que merece un fenómeno complejo que tiende a escaparse del marco analítico convencional de la sociología y la ciencia política.

Denigrar de entrada el populismo, adjetivarlo en negativo (Delsol 2015), no ayuda a su análisis y comprensión. Emplear la demagogia como significante equivalente y designar como populista a cualquier fuerza que represente un registro impugnatorio del orden dominante termina por "hacer circular el término" (Vallespín y Martínez-Bascuñán 2017) y debilita a la sociedad, cuando no cancela aquellas instancias de auto-reflexividad que le son propias.

El populismo es en el campo político, empleando una enunciación orteguiana, el tema de nuestro tiempo y, por mor de ello, ha de ser tratado con el mayor rigor analítico.

El populismo es un intruso para la ciencia social, en expresión de J. Alemán y G. Cano (2016), sobre todo para la denominada ideología neoliberal, con la que se relaciona, en una primera instancia, de forma antagónica como movimiento reactivo ante las consecuencias sociales de sus políticas. Un intruso con múltiples facetas que, además de ser reactivo, quiere ser en ocasiones un movimiento emancipador, caso del populismo de izquierdas, en contraste antagónico con el denominado populismo de derechas.

Sin embargo, el populismo nunca es un intruso, ya que esto equivaldría a interpretarlo como algo exterior a la democracia y que implica una amenaza potencial para sus fundamentos. Definirlo como ese exterior inquietante no es en absoluto contradictorio con la tesis de que el populismo es algo intrínseco o consustancial a la propia democracia (Rivero, Zarzalejos y del Palacio 2017). Su carácter de intruso y exterior se adquiere únicamente si es definido, paradójicamente en términos propiamente populistas, como un enemigo.
Si el populismo es un habitante siempre latente de la democracia, su análisis no puede reducirse de forma unilateral a comprender qué es, sino por qué retorna y cuál es su devenir.

La experiencia de los populismos en el mundo contemporáneo tiene sus raíces históricas a partir de los primeros procesos de modernización capitalista y la conformación de los Estados nacionales en el siglo $\mathrm{XIX}$.

El reciente ascenso de los populismos, a partir de la década de los años 70 del pasado siglo, se produce a partir del giro histórico que imprime la aceleración de la mundialización económica y de la caída de los regímenes comunistas. Este giro ha producido y sigue produciendo fracturas sociales y tensiones institucionales en los sistemas democráticos que se expresan bajo la forma de protestas, movimientos sociales $y$, finalmente, partidos políticos reactivos contra las limitaciones de los sistemas democráticos para dar respuestas efectivas a complejas demandas sociales y políticas.

En este contexto social y político de ascenso de los populismos, esta nota de investigación se plantea dos objetivos: en primer lugar, describir y analizar sumariamente tres libros recientes de autores nacionales en los que se aborda el fenómeno populista desde enfoques en los que el populismo se concibe, respectivamente, como una amenaza a la democracia (Rivero, Zarzalejos y del Palacio 2017), como síntoma de quiebra de la Ilustración (Lasalle, 2017) o como problema y reto a la vez para el sistema liberal-parlamentario (Vallespín y Martínez-Bascuñán, 2017) (sección 2); en segundo lugar, destacamos algunas limitaciones de estos enfoques analizados y, con base en ello, se sugiere un marco metodológico básico para poder aproximarse a la fenomenología populista, a la comprensión de sus aporías (sección 3).

\section{DEBATES RECIENTES SOBRE LOS POPULISMOS EN ESPAÑA}

Hemos escogido tres textos como objeto de revisión crítica. Dos de ellos, aunque con aproximaciones diferentes (Rivero, Zarzalejos y Del Palacio 2017; Lassalle 2017), forman parte de un enfoque neoliberal del populismo. El tercer texto (Vallespín y Martínez-Bascuñán 2017) entronca con un enfoque que cabe denominar social-liberal o socialdemócrata. Sintetizamos a continuación dichas aportaciones a la vez que destacamos algunos de sus límites.

El texto que coordina Rivero, junto con Zarzalejos y del Palacio, es un texto de naturaleza académica a la vez que de divulgación científica que, con veintinueve capítulos, aspira a ser "una geografía del populismo y portavoz de la academia internacional en lengua española”. 
En la introducción y en los tres capítulos de la primera parte, así como en el de conclusiones, los autores caracterizan el populismo por un triple rasgo: es consustancial a la democracia y emerge en momentos históricos de malestar; es, a la vez, un enemigo de la misma, a la que quiere destruir; y, de aplicarse, no es sostenible allí donde existen instituciones democráticas que hacen de contrapeso a su supuesta pobreza argumental e intensa emocionalidad.

Es de apreciar el esfuerzo que se hace al recorrer la geografía de los populismos, si bien el estilo de flashes analíticos no hace justicia a casos como el español. Así, la experiencia del 15-M y de Podemos se despachan en seis escasas páginas, además de analizarla junto al fenómeno del gilismo (capítulo 18). Lo mismo sucede con el capítulo 19 sobre nacionalismo y populismo. En la parte II no se hace mención alguna del populismo de Costa, como populismo originario sin base social.

El texto define el populismo, a modo de metáfora sanitaria, como una patología, como un fenómeno que "hablando la lengua de la democracia intenta acaba con ella". Como patología "endémica" se reconoce su permanencia, abierta o latente, en el seno de los sistemas democráticos. En este punto vienen a coincidir, desde enfoque extremos, con los planteamientos de Laclau y Mouffe (1987), para quienes el populismo tiene mayor probabilidad de aparecer en contextos donde la lógica institucional democrática no es capaz de satisfacer una amplia heterogeneidad de demandas sociales. Por tanto, es una posibilidad siempre abierta y presente. La diferencia estriba en que, para los primeros, el populismo es una anomalía institucional y, para los segundos, una realidad política intrínseca a la democracia.

El populismo, para Rivero, Zarzalejos y del Palacio, es una ideología con entidad propia. $\mathrm{Ni}$ es un conglomerado ideológico ni es sólo una "lógica de acción política", tal como sostienen Vallespín y Martínez-Bascuñán². Para los autores, la ideología no puede estar nunca ausente de un fenómeno como el del populismo, por muy confusa y pobre que se considere su argumentación.

Con base en esta caracterización general, los autores definen el populismo o los populismos por rasgos como los siguientes: la visión de un pueblo virtuoso, orgánico, con voluntad única; su consideración como movimientos en contra del sistema representativo liberal; el supuesto de que todo populismo, en su construcción de la política en el eje arriba/abajo, condena a las sociedades a un enfrentamiento fratricida que termina finalmente apelando a la aniquilación de "Ios enemigos del pueblo"; la inevitabilidad de un líder carismático autoritario trascendiendo la historia, o la necesidad de un enemigo permanente (política de enemistad al decir de Marzolf y Ganuza, [2016]).

Esta caracterización del populismo deja sin responder preguntas cruciales como son: si existen o pueden existir populismos que acepten la integración en el sistema democrático pluralista; si los movimientos sociales y partidos populistas, más que expresiones contra la democracia parlamentaria, representan una crítica de su funcionamiento (el no nos representan) y, por tanto, apelan a su perfeccionamiento y no a su destrucción, o si el eje antagónico de arriba/ abajo, más que dividir artificialmente a la sociedad, es un modo de articular una amplia hegemonía interclasista que limite el sobre-poder económico que los mercados financieros han ampliado e intensificado sobre los Estados democráticos. Preguntas todas ellas que no se plantean en el libro en cuestión.

Con base en este esquema doctrinal, los autores recomiendan tratar los populismos como una epidemia política a combatir en el plano ideológico. En este sentido, enfatizan que el populismo, como el liberalismo u otras opciones políticas, se basa en ideas y valores que deben ser combatidos. No es sólo un estilo de acción política, es una concepción de la política.

El texto de Lassalle es más un ensayo de debate político que una investigación stricto sensu. A pesar de su declarada militancia antipopulista, no deja de ser un texto con fundamento teórico e ideológico. Se posiciona contra el populismo como un fenómeno que representa un totalitarismo de tipo posmoderno, que opera como un exterior amenazante de la democracia. Coincide con Rivero, Zarzalejos y del Palacio en que el populismo es la causa por excelencia de la crisis de la democracia. Es un fenómeno recurrente que apela a la demagogia como forma de corrupción del pueblo. La equivalencia indiscutible entre populismo y demagogia se da por descontada, no necesita justificación.

El populismo vendría a ser, según Lassalle, un proto-totalitarismo tardo-moderno. Es más que un momento iliberal: es un fenómeno antiilustrado, radicalmente contrario a los fundamentos materiales y espirituales de la modernidad ilustrada. El populismo ha venido a enterrar la llustración y su pilar: la razón ilustrada, a la que opone la pasión populista. Una diferenciación que no parece tener en cuenta las consideraciones de otros teóricos del populismo que sostienen que "la razón es un bien escaso" en política (Villacañas 2015).

La calificación de la razón populista como razón pasional también permea paradójicamente su texto, al provocar el miedo con el supuesto de que "repta silenciosa y oculta a los ojos de la opinión pública la serpiente de un populismo que puede convertirse en la columna vertebral de un nuevo leviatán totalitario". Para Lasalle, la causa del populismo no reside en la corrupción institucional o en la gestión neoliberal de la gran depresión de 2008, estos son sólo sus catalizadores.

En sus propias palabras: "para entender el proceso populista hay que hurgar en la estructura emocio- 
nal de las sociedades occidentales y en el colapso de la ilustración". El 11-S y la gran depresión aceleran este proceso mediante el retorno del miedo y la inseguridad que provoca "la resaca emocional de saberse desposeído de las conquistas del bienestar y de derechos". En este punto converge con aquellos análisis que interpretan que las consecuencias de la crisis financiera han abonado el campo para el momento populista, caso de Vallespín y MartínezBascuñán (2017). Para Lassalle, el populismo es el retorno a una "modernidad regresiva" a la que es necesario combatir.

En el análisis de Lassalle están ausentes la dimensión del poder económico, los efectos de la nueva fase de la mundialización, los propios límites de los sistemas democráticos liberales para conciliar legitimidad y satisfacción de demandas ciudadanas y, no menos importante, la crisis de representatividad política, lo que contrasta con su visión elitista de la democracia cuando afirma que debemos "prevenirnos frente a los riesgos que aloja la arquitectura igualitaria y masiva de la democracia”. El autor no discrimina entre populismos ya que todos ellos, de derechas o de izquierdas, pluralistas o no, se reducen a la misma esencia: constituyen un peligro totalitario.

"Populismos", de Vallespín y Martínez-Bascuñán (2017), es un trabajo realizado desde la perspectiva de la ciencia política que sigue el proceso canónico de la investigación social.

Esta investigación tiene una relativa relación de vecindad con los anteriores trabajos en cuanto a que el populismo constituye una amenaza política para los sistemas liberal-parlamentarios. El populismo es, también aquí, un fenómeno preocupante: el afuera constitutivo $^{3}$ de la democracia liberal. Los autores, parafraseando a Marx, lo describen como el "espectro que recorre la democracia".

La relación de complementariedad entre democracia y populismo no parece posible para estos autores. En la senda de Müller (2016), consideran que existe una relación antagónica entre ambos ${ }^{4}$. Aquellos enfoques teóricos que inician su reflexión desde premisas basadas en el pluralismo de valores y la división de poderes (quintaesencia del liberalismo) no están reñidos con la posibilidad de realizar un análisis sobre el populismo. Sin embargo, a la hora de responder sobre la relación entre ambos fenómenos, dichas premisas ético-liberales conducen a que ineluctablemente la balanza valorativa se incline por contemplar (siempre) el populismo como un problema.

Esta obra no es una excepción. Lanza una advertencia sobre los peligros del populismo. Pero, además, plantea como solución a la crisis de la democracia, una salida en clave cosmopolita que aboga por generar una mejor gobernanza supra-nacional con capacidad para regular los procesos de integra- ción e interdependencia institucional y económica. Quiere alejarse de miradas nostálgicas e insulares y, en su crítica a esa pulsión matricial, al placer consolador de lo pretérito que muestran diferentes populismos, opone una solución que acepte el presente (principio de realidad) en toda su complejidad para poder seguir transitando hacia un futuro democrático abierto y plural.

Sin embargo, el hecho de compartir con los autores anteriores un profundo recelo sobre la naturaleza y fines del populismo no les convierte en lo mismo. Hay, invirtiendo una máxima de Heidegger, "distancia en la proximidad". La más notable es, sin duda, su análisis de las causas del populismo: el porqué, aunque también existen diferencias en el qué.

Los autores sostienen que el populismo debe ser concebido como un síntoma o síndrome. No es una patología de la democracia sino un fenómeno que nos revela dramáticamente una realidad social en tensión. En este punto, se aproximan a la interpretación del fenómeno populista como reacción social frente a los profundos cambios que tienen lugar en el actual proceso de modernización capitalista a nivel mundial y nacional.

Hoy, como señalan Vallespín y MartínezBascuñán, el principal problema es la descompensación que tiene lugar en la relación entre los requerimientos de eficiencia del capital y las demandas de justicia social. El mercado, la oligarquía de las finanzas, ha roto el pacto social que vertebraba el consenso europeo desde la posguerra (en línea con lo afirmado por Streeck [2017]). A diferencia de Rivero, Zarzalejos y del Palacio, y de Lassalle, los autores consideran que nos encontramos ante un poder económico sin constricciones, que coloniza el poder político, que desatiende las demandas sociales de igualdad. Acompañando a este proceso, no como relación causa-efecto, sino correlacionado con el mismo, aparecen otros factores no menos importantes como son: la crisis de representación política y la preeminencia de gobiernos técnicos o tecnócratas; las dificultades de crear espacios de consenso en un mundo de comunicación fragmentada, y, no menos importante, la crisis cultural como pérdida de referentes que favorecen los nacionalismos y la anomia individualista.

Siguiendo la senda del libro El gran retroceso (Nachtwey 2017), los autores consideran que estamos en un fin de época ante la que el populismo emerge como reacción nostálgica. Es la entrada en la sociedad del descenso la que trae consigo una parálisis de la movilidad social ascendente y el acceso a una nueva fase histórica de insatisfacción de las demandas sociales por el sistema democrático. Demandas que se ven bloqueadas por la descompensación que se ha producido entre mercado y democracia y por la incapacidad creciente de mediación del Estado. 
Los autores marcan con claridad la diferencia entre su enfoque y los de naturaleza neoliberal y populista. Para ellos, los partidos del establishment o sistémicos han adoptado una posición tecnocrática, afín a la hipótesis de la post-política (Zizek 2010), en la cual la dominación neoliberal es interiorizada como principio de realidad y los imperativos del mercado se viven como destino ineludible. Por otro lado, el populismo reacciona prometeicamente, ignorando u obviando los constreñimientos macro-estructurales de economías y sistemas cada vez más interdependientes.

La definición de qué sea populismo conduce a denominarlo como un ejemplo de síndrome de cenicienta (Berlín 1969), como metáfora de la tensión no resuelta entre definiciones que basculan entre el extremo genérico y el esencialismo. Por ello, los autores señalan que es posible esbozar tres vías de acceso al concepto de populismo: definirlo a partir de un tipo ideal, al modo weberiano; asumir una amplia heterogeneidad sin un criterio conceptual que lo discrimine, o, con base en una definición minimalista fundada en unos pocos axiomas, en línea con los autores del denominado "enfoque ideacional" (Hawkins y Rovira Kaltwasser 2017).

Los autores optan por el primer enfoque, siguiendo el trabajo clásico de lonescu y Gellner (1969) o el más reciente de Moffit (2016), contemplando diferentes niveles de análisis como son ideología, estrategia, retórica y lógica de acción política. Este último nivel o rasgo es el que mejor define la naturaleza del populismo, un fenómeno sin ideología representativa o no identificable, o débil. El populismo responde, sobre todo, a una lógica política de calculada ambigüedad instrumental. No es un proyecto ideológico sino una técnica de acceso al poder. Su ideología es débil o dispersa.

A partir de estos supuestos, el populismo es una reacción que, bajo la forma retórica del desasosiego, apela a soldar una comunidad sacrificando el pluralismo político. No queda claro en el texto por qué el populismo se puede reducir a una lógica de acceso al poder basada en la simplificación retórica de mensajes, ni por qué no puede también ser una posible condensación de diferentes enfoques teóricos, por muy confusos y fragmentados que se presenten.

Finalmente, el populismo entrañaría para los autores una guerra de representaciones, más que de ideologías, que tiende a minar el pluralismo político debido a su simplificación argumental y a su estrategia de polarización política.

\section{LA COMPLEJIDAD ANALÍTICA DEL FENÓMENO POPULISTA}

Los populismos constituyen un fenómeno global, pero experimentado en los espacios nacionales, lo cual modula de manera particular la constitución de sus bases sociales, sus estrategias políticas y sus retóricas ideológicas (Moffit 2016) y, por tanto, sus salidas políticas concretas y sus consecuencias institucionales, tanto para el liberalismo democrático en particular, como para la democracia en general.

Podemos definir tentativamente el populismo o populismos como: fenómenos socio-políticos reactivos a situaciones sociales y políticas de alta incertidumbre, de relativa amplia base social, fundados en torno a referentes simbólicos; cuyas demandas, tan amplias como difusas, son canalizadas a través de partidos u organizaciones, con mayor o menor densidad carismática, a través de los cuales se construyen discursos, identidades y retóricas dirigidas a la defensa de un statu quo social o nacional que se considera perdido, así como al rechazo antagonista de aquellas elites o grupos, nacionales y/o extranjeros, a los que se considera enemigos de un pueblo o comunidad nacional.

Definida la naturaleza del populismo como patología política (Rivero, Zarzalejo y del Palacio), como totalitarismo anti-ilustrado de baja intensidad (Lassalle) o como lógica de acción política iliberal (Vallespín y Martínez Bascuñán), el resultado es que, con importantes variantes, todos ellos muestran limitaciones metodológicas para una adecuada comprensión del populismo. Todos estos trabajos tienen su déficit en el análisis de la dimensión socio-histórica del populismo y en un excesivo énfasis en el análisis de impacto en el sistema democrático. Sólo el texto de Vallespín y Martínez-Bascuñán se aproxima con una visión amplia a la comprensión de la naturaleza del fenómeno y a sus complejas raíces socioculturales y económicas.

Los tres textos aquí resumidos son una muestra, ciertamente limitada, de que las aporías del populismo y sus ambivalencias políticas nos indican, de entrada, la enorme dificultad para alcanzar consensos metodológicos que favorezcan un análisis fructífero.

La génesis socio-histórica ${ }^{5}$ de los populismos o, si se prefiere, sus fundamentos sociales, son quizás la mayor debilidad de los trabajos analizados. El análisis social requiere la consideración de tres procesos de cambio que están en la base de los populismos. Si bien no son procesos que actúen automáticamente, sí operan como condición de posibilidad de los mismos ${ }^{6}$ : los procesos de "dualización" y desigualdad social provocados por el cambio socio-técnico; el retroceso, cuando no regresión, del estado de bienestar como institución de mediación entre mercado y democracia, necesaria para la creación de cohesión social y espacial y, por tanto, la falta de capacidad para moderar dichos procesos de "dualización", y, finalmente, la percepción ciudadana de que los sistemas democráticos no pueden satisfacer buena parte de su propia oferta, la canalizada a través del sistema de partidos en competencia. 
La preferencia por el análisis del posible impacto corrosivo del populismo en los sistemas de democracia liberal representativa sitúa, de antemano, el análisis en una dimensión moral que puede hacer perder de vista una pregunta decisiva, aquella que interroga por las causas estructurales de fondo que están en el origen del conflicto que constituye la fuente de la situación populista.

Entendemos que esto reduce el curso de un análisis que demanda diferenciar varios planos o niveles analíticos. Por una parte, todo análisis de los populismos tiene que tomar en consideración los fundamentos sociales, políticos y económicos, tal y como plantea Judis (2016). Se trata de pensar las condiciones estructurales de posibilidad de emergencia del sujeto populista, es decir, aquellas condiciones que, sin determinar, favorecen la posibilidad del populismo. Como sugiere E. Laclau (2016), estos efectos "dislocatorios" en el nivel de la estructura tienen siempre como efecto la quiebra del imaginario simbólico donde descansa el orden social o, lo que es lo mismo, una crisis de legitimidad aguda, condición necesaria, aunque no suficiente, para el retorno del populismo.

Una segunda dimensión analítica consiste en pensar los propios actores populistas. Esto es, el tipo de revueltas, movimientos sociales o partidos que son expresiones populistas, de tal forma que se pueda brindar una explicación no sólo sobre sus distintas tipologías o fisonomías, sino por qué, cómo y cuándo se forman, así como los factores que explican por qué pueden diluirse. Entendiendo que la excepcionalidad, o tiempo límite, es el contexto en que aparecen, la clausura o cierre de esa herida social puede socavar el núcleo traumático de emergencia. Es decir, si parte de las demandas sociales son satisfechas institucionalmente, el populismo pierde el malestar social, difuso y heterogéneo, que le abrió la ventana de oportunidad.

Por último, es necesario analizar su despliegue real en la arena política, es decir, analizar sus acciones en su más amplio sentido. Nos referimos de forma concreta al análisis del discurso, el estilo político y el tipo de liderazgo.

Cabe destacar, por su importancia, algunas limitaciones vinculadas al tercer nivel analítico. Nos referimos a la cuestión de la ideología, punto ciego del populismo. Es posible preguntarse si el populismo, en sus distintas manifestaciones históricas y espaciales, posee una ideología identificable. La respuesta depende en gran medida de la definición que utilicemos. En todo caso, sin poder reducir el fenómeno a una sola ideología concreta, como sostienen acertadamente Laclau (2016), Vallespín y Martínez-Bascuñán (2017) o Moffit (2016), o a unos pocos axiomas, como plantean Rivero, Zarzalejos y del Palacio (2017), no podemos decir que el populismo sea un fenómeno sin ideología, es decir, sin principios, valores y objetivos ${ }^{7}$, por más que acontezcan de forma difusa, ambigua y heterogénea.
Es necesario analizar la expresión ideológica concreta del populismo, aunque sea confusa y contradictoria, como se corresponde con reacciones sociales de protesta naturaleza interclasista. Más que de ideología débil o simplificada, cabe calificarla de conglomerado ideológico; un entreverado que se corresponde tanto con la construcción de una cosmovisión anti-oligárquica de amplia base social como de una semántica comunitarista. Lo que Ortí (1988) denomina discurso populista básico.

Los tres trabajos analizados ponen el énfasis en la retórica del populismo, en su discurso, al que de manera conjunta caracterizan de simplificador. La retórica populista sería simple ambigüedad calculada. Un mecanismo instrumental de seducción demagógica y maniquea de las masas. Sin embargo, esta aproximación plantea algunos problemas. En primer lugar, el discurso simplificador no es un rasgo exclusivo de los populismos, sino que es una realidad que atraviesa a la totalidad del conjunto de fuerzas políticas, máxime en un contexto político donde las estructuras de oportunidad comunicativas favorecen estilos simplificados y emocionales.

Por otro lado, la retórica no puede reducirse únicamente a un juego semántico de apariencias, sino que, como plantea Laclau (2016), el discurso es ontológicamente constitutivo de lo social. Es decir, desde este enfoque constructivista se afirmaría que el populismo es un nominalismo o, lo que es lo mismo, que el pueblo es una construcción nominativa.

Por último, como modo de evitar la reducción de los populismos a retórica política, los discursos populistas serían el instrumento de comunicación que enlaza, mejor o peor, la base social con los referentes simbólicos y la ideología. Pero, con carácter previo, es exigible el análisis de su base social y su ideología difusa constitutiva.

La construcción social y política de los populismos no anuncia una alternativa única. Un problema a discernir es si las reacciones populistas, en el caso europeo, lo son contra las políticas neoliberales predominantes en las últimas décadas o contra el propio sistema democrático. Los textos analizados se sitúan, más bien, en esta última posición y no parecen contemplar que los populismos reactivos pueden acabar en formas de reintegración política democrática, bajo formas de recentralización del poder existente, o, simplemente, diluyéndose.

\section{Conclusiones}

Esta nota de investigación analiza tres trabajos de análisis y debate sobre los populismos que fundan su esencia en ser, respectivamente, una epidemia política (Rivero, Zarzalejos y del Palacio), una amenaza para el porvenir de la ilustración (Lassalle) o una lógica de acción política que puede hacer pe- 
ligrar el sistema democrático (Vallespín y MartínezBascuñán). En síntesis, el populismo se define como una amenaza a los regímenes demo-liberales.

En el primer caso, el populismo es algo intrínseco a la democracia, un fenómeno que habita en latencia y que retorna bajo determinadas condiciones. Para Lassalle, un celo liberal-elitista le invita a pensarlo como una amenaza interna propia de la lógica igualitaria de la democracia de masas. En cambio, para Vallespín y Martínez-Bascuñán, responde más bien a una mutación económica, social, política y cultural de las democracias avanzadas que provoca una lógica de acción política que puede hacer peligrar el curso del desarrollo democrático.

De este análisis, necesariamente sintético, planteamos la necesidad de abordar el análisis de los populismos no como intrusos de la democracia sino como un problema social relevante, que es tanto síntoma de crisis sociales profundas como un reto de profundización de la democracia.

Si este planteamiento es correcto será necesario abordar de manera integrada todos los niveles y factores que exige un análisis tan complejo. Es decir, el de

\section{NOTAS}

1. La idea de límite está tomada del filósofo Eugenio Trías (2006).

2. F. Vallespín y M. M. Bascuñán toman el concepto de lógica de acción política de E. Laclau (2016).

3. Término que toma Laclau (2016) de J. Lacan.

4. Moffit (2016) señala tres formas de concebir la relación o impacto del populismo sobre la democracia: negativamente, positivamente o de forma agnóstica.

\section{REFERENCIAS BibLIOGRÁficAS}

Alemán, J. y G. Cano. 2016. Del desencanto al populismo: Encrucijada de una época. Barcelona: NED.

Berlin, I. 1969. To Define Populism. The Isaiah Berlin Virtual Library.

Delsol, C. 2015. Populismo: una defensa de lo indeseable. Madrid: Ariel.

Hawkins, K. y C. Rovira Kaltwasser. 2017. The ideational approach to populism. Latin American Research Review 52 (4): 513-528.

Ionescu, G. y E. Gellner (Ed.). 1969. Populism: its meaning and national characteristics. London: Weidenfeld \& Nicholsen.

Judis, J. B. 2016. The populist explosion: How the great recession transformed American and European Politics. Nueva York: Columbia Global Reports.

Laclau, E. 2016. La razón populista. Madrid: Fondo de Cultura Económica.

Laclau, E. y C. Mouffe. 1987. Hegemonía y estrategia socialista. Hacia una radicalización de la democracia. Madrid: Siglo XXI.

Lassalle, J.M. 2017. Contra el populismo. Madrid: Debate.

Marzolf, H. y E. Ganuza. 2016. “¿Enemigos o colegas? El $15 \mathrm{M}$ y la hipótesis Podemos". EMPIRIA. Revista de Metodología de Ciencias Sociales 33: 89-110. la naturaleza de los actuales procesos de modernización económica y socio-técnica en el marco de la globalización neoliberal, la crisis del Estado de Bienestar y el bloqueo de la satisfacción de derechos sociales y expectativas de participación política; así como, no menos importante, la crisis cultural e ideológica provocada por el choque de valores e ideas entre un campo en retroceso que defiende modos de cohesión social y otro dominante de individualismo posesivo.

El análisis de los populismos exige ir más allá de su reducción a la demagogia y a vacíos constructos retóricos para, con mayor rigor y, sin duda, incertidumbre, articular los diferentes y necesarios planos de análisis antes mencionados. Es decir, la consideración de aquellos cambios socio-económicos profundos que engendran una crisis de legitimidad en una sociedad, que se expresan en momentos populistas de desafección política, temor y extensa incertidumbre. También es conveniente considerar las condiciones políticas y culturales que canalizan la desafección por los más variados cauces que, en general, suelen ser reactivos, identitarios o de repliegue (los más) y defensivos, integradores y cosmopolitas (la excepción).

5. Entendemos por análisis socio-histórico aquel método que analiza el presente a partir de ese pasado que le precede y que lo preforma y sienta las condiciones de su desarrollo ulterior.

6. Nos referimos fundamentalmente al contexto europeo.

7. En un libro reciente, Sánchez-Cuenca (2018), plantea estos tres criterios para definir qué es una ideología frente a explicaciones funcionalistas o basadas en la formación de ideas.

Moffit, B. 2016. The global rise of populism: Performance, political style and representation. Stanford CA: Stanford University Press.

Mudde, C. y C. Rovira Kaltwasser. 2017. Populism: A very short introduction. New York, NY: Oxford University Press.

Müller, J-W. 2016. Qué es populismo? Ciudad de Méjico: Grano de sal.

Nachtwey, O. 2017. La sociedad del descenso: Precariedad y desigualdad en la era posdemocrática. Barcelona: Paidós.

Ortí, A. 1988. "Para analizar el populismo: movimiento, ideología y discurso populistas". Historia Social 2: 75-98.

Rivero, A., J. Zarzalejos y J. del Palacio. 2017. Geografía del populismo. Madrid: FAES.

Sánchez-Cuenca, I. 2018. La superioridad moral de la izquierda. Madrid: Colección Contextos.

Streeck, W. 2017. ¿Cómo terminará el capitalismo? Madrid: Traficantes de Sueños.

Trías, E. 2006. La política y su sombra. Barcelona: Anagrama.

Vallespin, F. y M. Martínez-Bascuñan. 2017. Populismos. Madrid: Alianza.

Villacañas, J.L. 2015. Populismo. Madrid: La Huerta Grande.

Zizek, S. 2010. En defensa de la intolerancia. Madrid: Público. 
ARTURO RODRÍGUEZ SÁEZ. Licenciado en Ciencias Políticas y de la Administración por la UCM (2012) y máster en Democracia y Gobierno por la UAM (2014), actualmente es doctorando en el Programa de Doctorado de Sociología y Antropología de la Universidad Complutense de Madrid y realiza su tesis doctoral sobre el populismo de izquierdas en España. Ha colaborado en diferentes proyectos de investigación. Sus áreas de investigación e interés se centran en los movimientos sociales, el uso político de los nuevos medios de comunicación, las políticas sociales, el populismo y la teoría política. 